\title{
Sustained release of vancomycin from novel biodegradable nanofiber-loaded vascular prosthetic grafts: in vitro and in vivo study
}

This article was published in the following Dove Press journal:

International Journal of Nanomedicine

29 January 2015

Number of times this article has been viewed

\author{
Kuo-Sheng Liu' \\ Cheng-Hung Lee ${ }^{2}$ \\ Yi-Chuan Wang ${ }^{3}$ \\ Shih-Jung Liu ${ }^{3}$ \\ 'Department of Thoracic and \\ Cardiovascular Surgery, Chang Gung \\ Memorial Hospital, Linkou, Taiwan; \\ ${ }^{2}$ Division of Cardiology, Department \\ of Internal Medicine, Chang \\ Gung Memorial Hospital, Linkou, \\ Taiwan; ${ }^{3}$ Department of Mechanical \\ Engineering, Chang Gung University, \\ Tao-Yuan, Taiwan
}

\begin{abstract}
This study describes novel biodegradable, drug-eluting nanofiber-loaded vascular prosthetic grafts that provide local and sustained delivery of vancomycin to surrounding tissues. Biodegradable nanofibers were prepared by first dissolving poly $(\mathrm{D}, \mathrm{L})$-lactide-co-glycolide and vancomycin in 1,1,1,3,3,3-hexafluoro-2-propanol. The solution was then electrospun into nanofibers onto the surface of vascular prostheses. The in vitro release rates of the pharmaceutical from the nanofiber-loaded prostheses was characterized using an elution method and a highperformance liquid chromatography assay. Experimental results indicated that the drug-eluting prosthetic grafts released high concentrations of vancomycin in vitro (well above the minimum inhibitory concentration) for more than 30 days. In addition, the in vivo release behavior of the drug-eluting grafts implanted in the subcutaneous pocket of rabbits was also documented. The drug-eluting grafts developed in this work have potential applications in assisting the treatment of vascular prosthesis infection and resisting reinfection when an infected graft is to be exchanged.
\end{abstract}

Keywords: drug-eluting prosthetic graft, vascular prosthesis infection, release characteristics

\section{Introduction}

Prosthetic graft infection is a major challenging complication in vascular surgery. Treatment of vascular prosthesis infection usually involves total or subtotal graft excision, radical debridement of the local infected tissue, and systemic antibiotics. ${ }^{1}$ However, the need for complete excision of an infected graft is sometimes counterbalanced by the more important need to provide vascular access for hemodialysis in a patient with limited access options. Replacing the infected graft with a new prosthesis carries a high risk of bacterial colonization and subsequent reinfection.

The systemic administration of antimicrobial medications by itself fails to treat vascular prosthesis infection, largely due to inadequate drug concentrations at the site of infection. Primary advantages of a drug-eluting graft are the higher local drug concentration and minimized systemic side effects. Therefore, we believe that the simultaneous combination of total or subtotal graft excision and a replacement with an antibiotic-eluting graft may provide a better possibility of infection control while maintaining the vascular access at the same time.

This study presents novel biodegradable, vancomycin-eluting nanofiber-loaded vascular prosthetic grafts for the local delivery of sustainable antibiotics. Biodegradable nanofibers were prepared by first dissolving poly(D,L)-lactide-co-glycolide (PLGA) and vancomycin in 1,1,1,3,3,3-hexafluoro-2-propanol (HFIP). The solutions were then electrospun onto the surface of commercially available vascular grafts. 


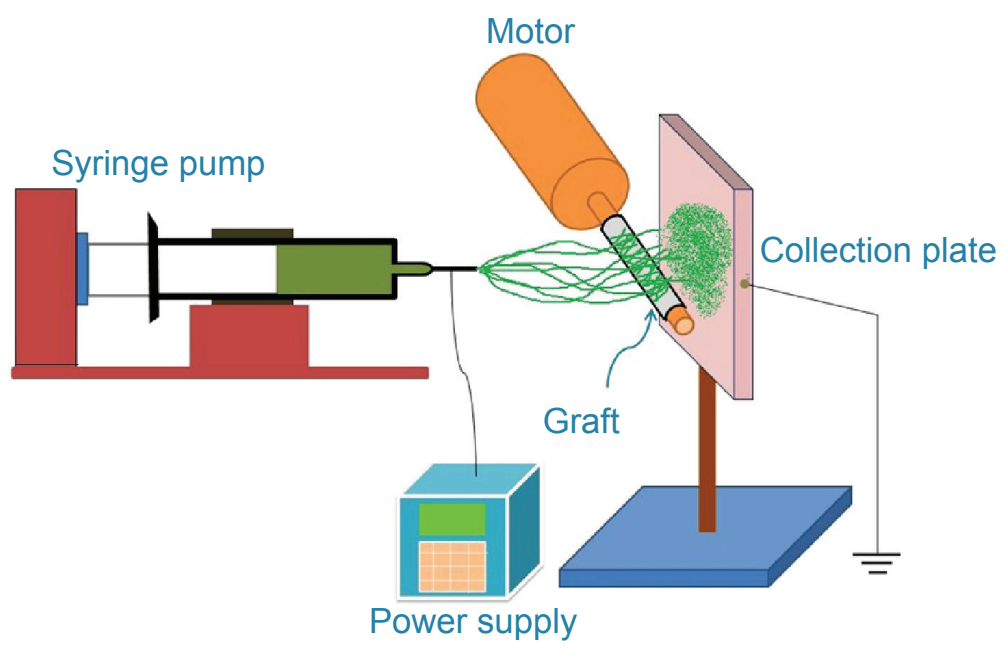

Figure I The electrospinning setup shown schematically.

After electrospinning, the morphology of electrospun nanofibers was characterized by a scanning electron microscope (SEM). The characteristics of the in vitro release of the pharmaceutical from the nanofibers were studied. Additionally, the in vivo delivery of antimicrobial vancomycin at and near the implanted site of rabbits was investigated. The systemic drug concentration in blood was also examined.

\section{Materials and method}

\section{Fabrication of drug-eluting grafts}

The PLGA used is commercially available material (Resomer RG 503; Boehringer Ingelheim GmbH, Ingelheim, Germany) and has a lactide:glycolide ratio of 50:50, and a molecular weight of 33,000 Da, which was measured by a gel permeation chromatograph equipped with a Waters 2414 Refractive Index Detector (Waters Corp, Milford, MA, USA). Vancomycin and HFIP were purchased from Sigma-Aldrich Corp (St Louis, MO, USA).

Biodegradable drug-eluting nanofibers were coated onto the external surface of prosthetic grafts (IMPRA ${ }^{\circledR} 6 \mathrm{~mm} \times 40 \mathrm{~cm}$; Bard Peripheral Vascular, Inc., Tempe, AZ, USA), using an electrospinning process. The nanofibers were prepared by first dissolving PLGA and vancomycin (240 mg and $40 \mathrm{mg}$, respectively) into $1 \mathrm{~mL}$ of HFIP. The solution was then delivered and electrospun by a syringe pump with a volumetric flow rate of $3.6 \mathrm{~mL} / \mathrm{hr}$ to create nanofibers, onto the prosthetic grafts. The electrospinning setup of this study consisted of a syringe and needle (internal diameter of $0.42 \mathrm{~mm}$ ), a ground electrode, a prosthetic graft mounted on a motor, a collection plate, and a high-voltage supply, as shown schematically in Figure 1. The needle was connected to the high-voltage supply, which generated positive direct current (DC) voltages and currents, up to $35 \mathrm{kV}$ and $4.16 \mathrm{~mA} / 125 \mathrm{~W}$, respectively. The rotational speed of the motor was $300 \mathrm{rpm}$. The distance between the needle tip and the ground electrode was $10 \mathrm{~cm}$, and the positive voltage applied to the polymer solutions was $17 \mathrm{kV}$. All electrospinning experiments were performed at room temperature. Figure 2 displays the electrospun nanofiber prosthetic grafts (top) and the bare grafts (bottom). All nanofiber-mounted prosthetic grafts were placed in a vacuum oven at $40^{\circ} \mathrm{C}$ for 72 hours for solvent evaporation.

\section{Standard curve of vancomycin}

The standard curve of drug concentrations was determined using a high-performance liquid chromatography (HPLC) assay. The HPLC analyses were conducted on a Hitachi L-2200 Multisolvent Delivery System (Hitachi Ltd., Tokyo, Japan). A Symmetry $\mathrm{C}_{8}, 3.9 \mathrm{~cm} \times 150 \mathrm{~mm}$ HPLC column (Waters Corp) was used for the separation of vancomycin. The mobile phase contained $0.01 \mathrm{~mol}$ heptanesulfonic acid

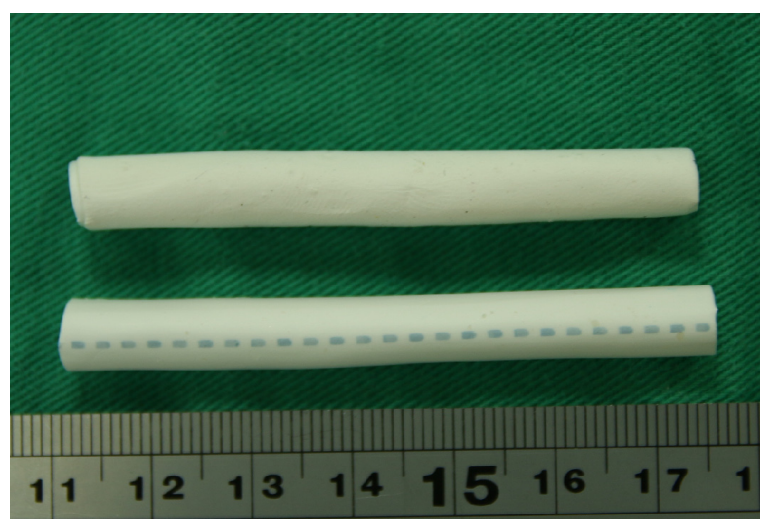

Figure 2 Photograph of the vascular prosthetic grafts (top, loaded with nanofibers; bottom, bare graft). 
(Thermo Fisher Scientific Inc., Waltham, MA, USA) and acetonitrile (Mallinckrodt Pharmaceuticals, Dublin, Ireland) $(85 / 15, v / v)$. The absorbency was monitored at a wavelength of $280 \mathrm{~nm}$, and the flow rate was $1.4 \mathrm{~mL} / \mathrm{min}$. All experiments were undertaken in triplicate, and the sample dilutions were performed to bring the unknown concentrations into the range of the assay standard curve. A calibration curve was made for each set of measurements (correlation coefficient $>0.99$ ). The elution product was identified and quantified with a high degree of sensitivity, using the HPLC system.

\section{In vitro release of pharmaceuticals}

The release characteristics of vancomycin from the drugeluting grafts were determined by the in vitro elution method. The vascular graft was cut into $2 \mathrm{~cm}$ long segments, which were then placed into glass test tubes (one sample per test tube $[\mathrm{n}=3])$ with $1 \mathrm{~mL}$ of phosphate buffer solution $(0.15 \mathrm{~mol} / \mathrm{L}$, $\mathrm{pH}$ 7.4) in each test tube. The glass test tubes were then incubated at $37^{\circ} \mathrm{C}$ for 24 hours before the eluent was collected and analyzed. Next, fresh phosphate buffer solution $(1 \mathrm{~mL})$ was added to each test tube for the next 24-hour period; this procedure was repeated for 35 days. Finally, the drug concentrations in the eluents were determined by the HPLC assay standard curve.

\section{In vivo study}

Fifteen New Zealand white rabbits with an average weight of $3.0 \mathrm{~kg}$ were used in the animal study. All animal procedures received institutional approval, and all studied animals were cared for in line with the regulations of the Ministry of Health and Welfare of Taiwan and under the supervision of a licensed veterinarian. During the surgical procedure, the animals were anesthetized with an intramuscular injection of $1 \mathrm{~mL}$ \% Rompun ${ }^{\circledR}$ (Bayer HealthCare, Leverkusen, Germany) and $1 \mathrm{~mL}$ Zoletil ${ }^{\circledR} 50$ (Virbac Laboratories, Carros, France). They maintained spontaneous breathing without intubation. Following anesthetization, a $2 \mathrm{~cm}$ skin incision was made in each rabbit's back. A subcutaneous pocket was made, into which the drug-eluting graft was inserted. The drugeluting graft was $2 \mathrm{~cm}$ in length. Inside the graft, a $5 \times 5 \mathrm{~mm}$ plain prosthetic patch (IMPRA; Bard Peripheral Vascular, Inc.) was placed. Both ends of the drug-eluting graft were sewn closed, using 5-0 polypropylene sutures, before its insertion into the animal so that the drug released into the surrounding tissue would not move to the inside of the graft. The animals were evenly divided into five groups. The implanted vascular grafts were taken out from the animals at postoperative weeks $1,2,3,4$, and 5. Tissue samples were obtained for vancomycin concentration measurement from just beneath the graft, $1 \mathrm{~cm}$ from the graft, and $2 \mathrm{~cm}$ from the graft. The plain prosthetic patch was also retrieved for drug concentration measurement, as a sample of drug elution from the nanofibers to the inside of the graft. Blood concentrations of vancomycin were collected by syringes via puncture on the marginal ear vein on postoperative days 1, 2, 3, 7, 14, 21, 28, and 35 . The animals received the same anesthetic protocol as for the surgical procedure before each venous sampling.

\section{SEM observation}

The morphology of the electrospun nanofibers was observed on an SEM (Hitachi S-3000N; Hitachi Ltd.), after gold coating.

\section{Results}

In this study, by adopting appropriate process parameters (eg, solvent, polymer concentration, and flow rate), vancomycin-loaded vascular prosthetic grafts were successfully fabricated using the electrospinning procedure (Figure 2). Figure 3 shows the SEM micrographs of the electrospun drug-eluting nanofibers (under a magnification of 3,000×). The diameters of the spun PLGA/vancomycin nanofibers ranged from 70 to $1,200 \mathrm{~nm}$. Additionally, the pore size of the nanofiber surface was approximately 5-10 $\mu \mathrm{m}$.

\section{In vitro elution of vancomycin from drug-eluting grafts}

Figure 4 plots the in vitro daily release curves of vancomycin. According to this graph, the drug-eluting grafts continuously released high concentrations of vancomycin (well above the $90 \%$ minimum inhibitory concentration $\left[\mathrm{MIC}_{90}\right]$ ) for more

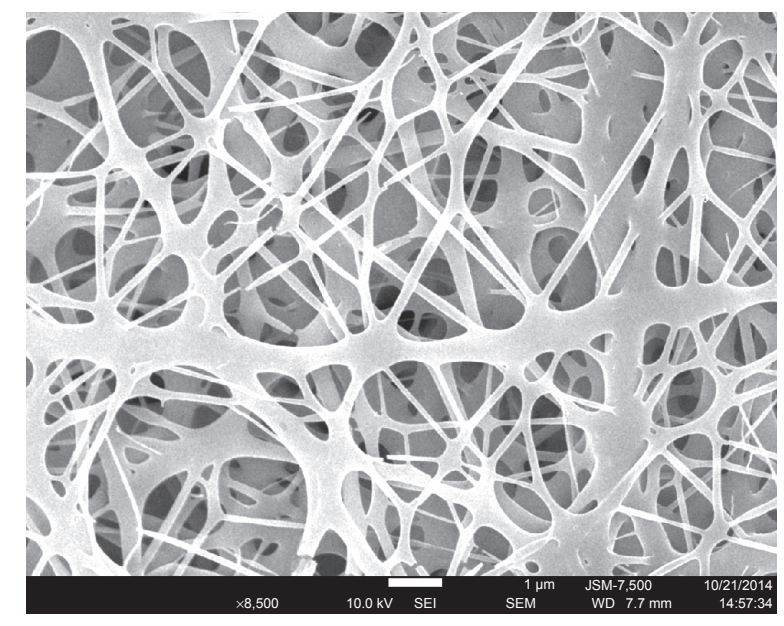

Figure 3 SEM photograph of electrospun drug-eluting nanofibers. Abbreviation: SEM, scanning electron microscope. 


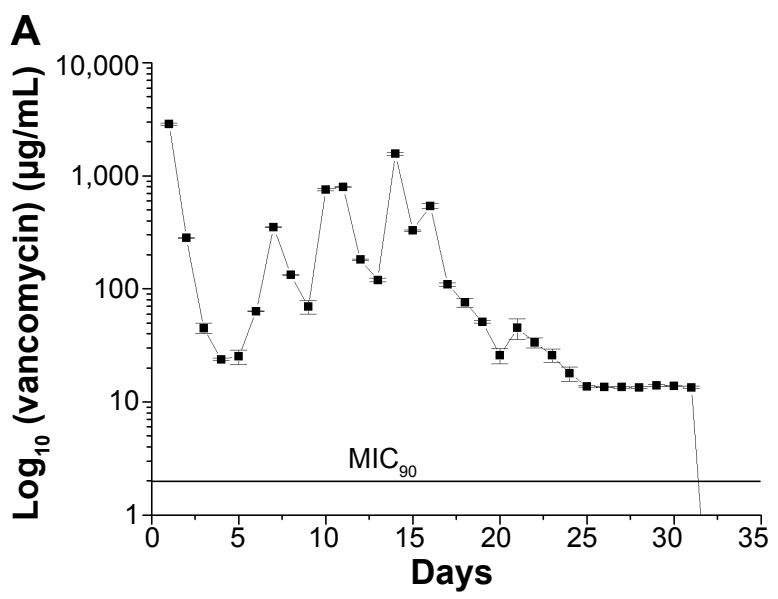

Figure $4 \mathrm{In}$ vitro release of vancomycin from the drug-eluting grafts $(n=3)$. Notes: (A) Daily release, and (B) accumulated release.

Abbreviation: $\mathrm{MIC}_{90}, 90 \%$ minimum inhibitory concentration.

than 30 days, with an initial burst period of 2 days, followed by peaks of release from days 7 to 15 , after which the concentration gradually decreased.

\section{In vivo release characteristics}

The vancomycin-eluting nanofiber-loaded graft was cut into grafts $2 \mathrm{~cm}$ in length. A subcutaneous pocket was made in each rabbit's back, into which the drug-eluting graft was inserted (Figure 5). The in vivo release characteristic of vancomycin from the graft was investigated. The systemic drug concentration in blood was also examined. Figure 6 shows the in vivo drug release at various days.

The drug concentrations right beneath the graft, $1 \mathrm{~cm}$ from the graft, and $2 \mathrm{~cm}$ from the graft all remained high for more than 5 weeks. Vancomycin concentration inside the graft was much lower than the concentration outside the graft. The graft itself seemed to act as a barrier so that the eluted vancomycin could not penetrate to the inside of the graft.
B

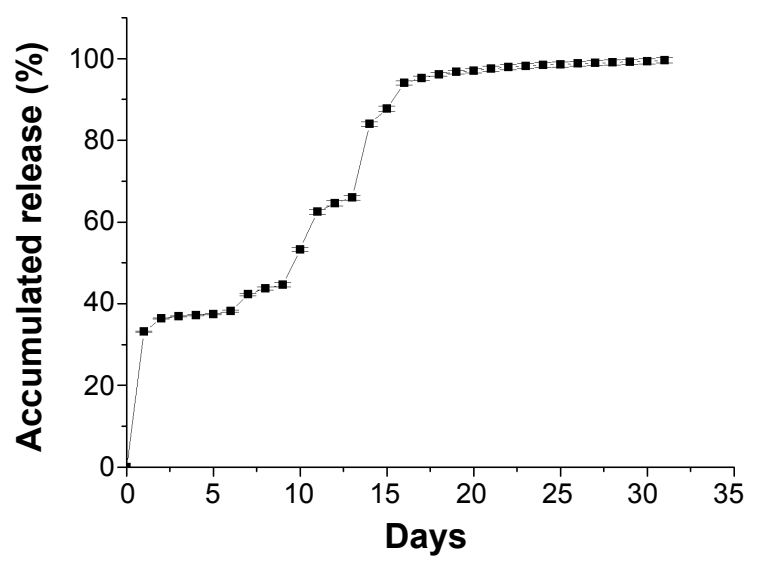

The blood concentration of vancomycin was also low throughout the study period.

\section{Discussion}

Vancomycin is an antibiotic useful for the treatment of a number of bacterial infections. It is of the glycopeptide antibiotic class and is effective mostly against gram-positive bacteria. Vancomycin acts by inhibiting proper cell wall synthesis in gram-positive bacteria. Due to the different mechanism by which gram-negative bacteria produce their cell walls and the various factors related to entry to the outer membrane of gram-negative organisms, vancomycin is not active against gram-negative bacteria (except some nongonococcal species of Neisseria). The pharmaceutical is primarily used for the treatment of serious infections caused by gram-positive bacteria known or suspected to be resistant to other antibiotics. The Infectious Disease Society of America recommends vancomycin as a first-line treatment for complicated
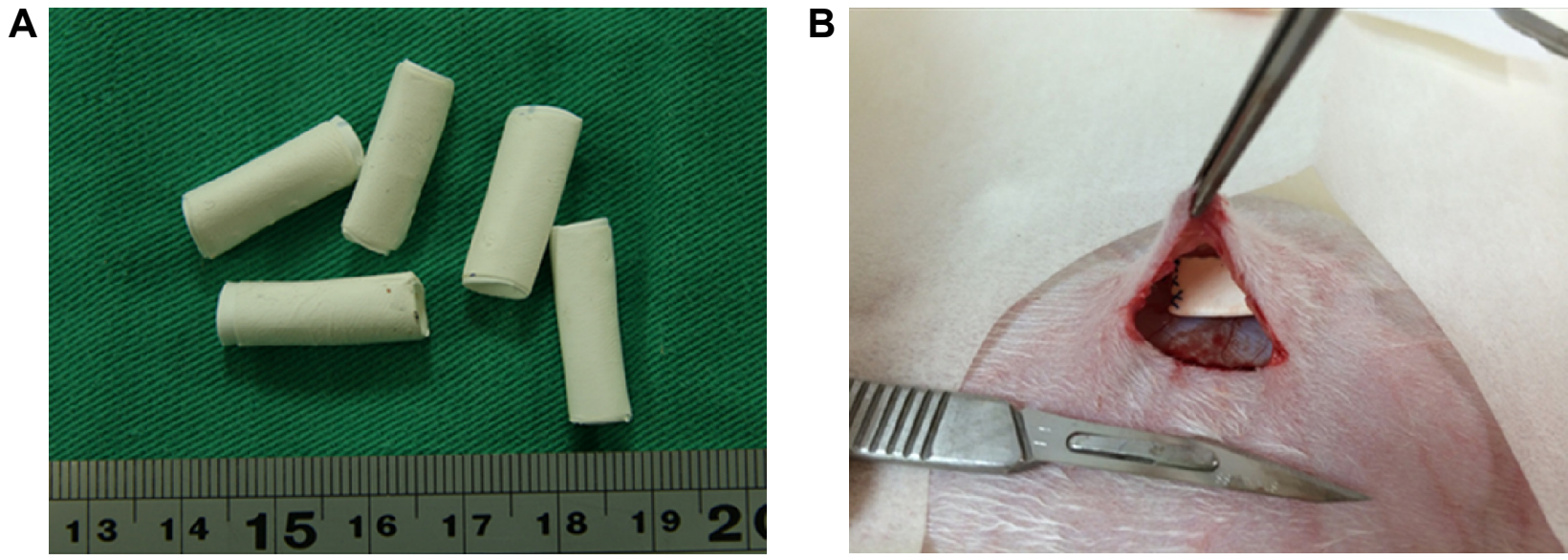

Figure $\mathbf{5}$ The implantation of drug-eluting grafts into the subcutaneous pockets of rabbits.

Notes: (A) The drug-eluting graft was cut into $2 \mathrm{~cm}$ in length. (B) The implantation of drug-eluting grafts into the subcutaneous pockets of rabbits. 


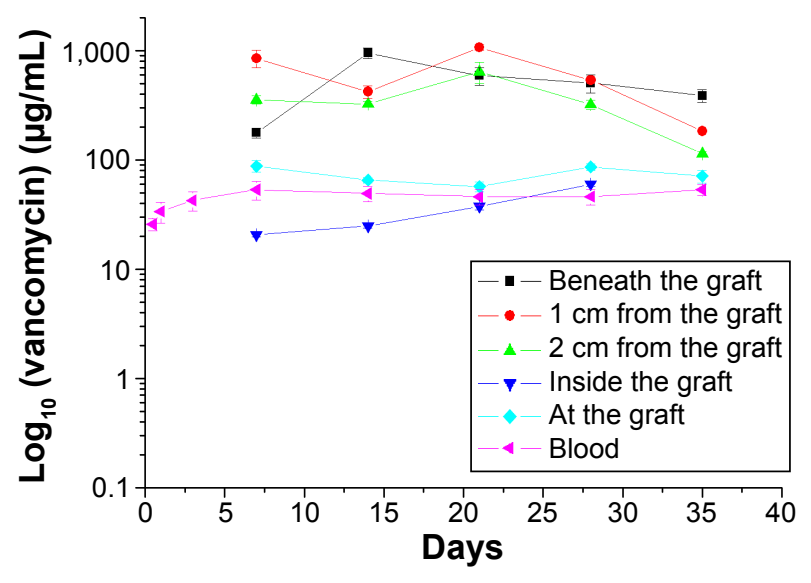

Figure 6 In vivo release behavior of the drug-eluting grafts.

skin infections, bloodstream infections, endocarditis, bone and joint infections, and meningitis infections caused by methicillin-resistant Staphylococcus aureus (MRSA). ${ }^{2}$

Infection is a common cause of arteriovenous (AV) graft loss. ${ }^{3,4}$ It has been found to account for about $35 \%$ of patients losing AV grafts. ${ }^{5}$ S. aureus is the leading cause of AV graft infection, ${ }^{6}$ and MRSA has emerged as the most prevalent pathogen. ${ }^{7}$ Evidence suggests that mortality associated with MRSA infection is much higher than mortality from methicillin-susceptible $S$. aureus infection..$^{8-11}$ Vancomycin has been the mainstay of parenteral therapy for MRSA infections. ${ }^{2,12}$ However, the systemic use of vancomycin is associated with increased nephrotoxicity and high-frequency hearing loss in older patients. ${ }^{13-15}$ Previously, systemic medication therapy was limited by the fixed pharmacokinetics of specific drugs. Combining drugs with a controlled-release method to create flexible hybrid delivery platforms (thereby decoupling chemical and kinetic properties) has revolutionized systemic therapy. Finding a way to increase vancomycin concentrations at the local tissue without adding risks of systemic toxicity is thus highly desirable.

Antibiotic-loaded polymethyl methacrylate (PMMA) beads have been successfully used for the treatment of extracavitary vascular site infections. ${ }^{7}$ However, the recurrent infection rate approached $20 \%$, and additional operations were required for bead change and eventually, bead removal. PMMA beads do not degrade in the human body and may serve as a potential nidus for bacterial colonization, causing recurrent infection. There have been studies on the use of antibiotic-impregnated or antibiotic-soaked grafts, but these, as the sole source of antibiotics, are often insufficient to prevent graft infection. ${ }^{16,17}$ Prophylactic rifampicin-soaked gelatin-impregnated grafts have failed to reduce graft infection rates at 1 month or at 2 years in a systematic review. ${ }^{18}$ Glues have also been used as a drug carrier for antibiotics, but the release periods they offered were less than 72 hours, ${ }^{19,20}$ which was inadequate for treatment of vascular graft infection.

This current study has developed biodegradable drugeluting PLGA nanofiber-loaded vascular prosthetic grafts by using the electrospinning scheme. Drug-eluting nanofibers can provide a sustainable release of high concentrations of vancomycin for more than 30 days in vitro, exhibiting triphasic release characteristics. After the electrospinning procedure, most pharmaceuticals were dispersed in the bulk of the PLGA matrix; however, some drugs might have been located on the surface of the nanofibers, subsequently leading to the initial burst of drug release. The initial burst is an undesired effect for sustained release. Recent studies demonstrated that the coating of the nanofibers with a blank biodegradable material could completely eliminate the initial burst effect. ${ }^{21,22}$ However, the initial antibiotic burst is favorable release kinetics for infection control because the exposure of bacterial microorganisms to a high starting dosage of antibiotics is important for bacterial eradication. ${ }^{23}$ Following the initial burst, drug release was controlled solely by the degradation of the polymeric materials. ${ }^{24}$ The nanofibers thus exhibited a rather stable release of a high concentration of vancomycin well above the $\mathrm{MIC}_{90}{ }^{25}$ of $2 \mu \mathrm{g} / \mathrm{mL}$, for more than 30 days. In vivo results also indicated that at 35 days, the vancomycin concentration was still high at the tissues beneath the graft and at $1 \mathrm{~cm}$ and $2 \mathrm{~cm}$ from the graft, while the drug concentration remained low at the graft itself and inside of the graft. This feature represents a significant advantage - achieving high vancomycin concentrations at the surrounding tissues of the graft while maintaining minimum systemic drug level, thus avoiding vancomycin-related adverse effects. Additionally, while the nanofibers could release a high concentration of vancomycin for only 32 days in vitro, the in vivo results indicated that the released vancomycin remained high even after 35 days. For all pharmaceuticals, the in vivo environment appears to provide a slower metabolic rate than the in vitro environment does. This phenomenon may explain why the effective period of in vivo drug release is longer than the period of the in vitro drug release.

Biodegradable polymers are a potential material for drug delivery as they can exist safely in the human body and can be eventually absorbed without causing harm or adverse interactions. The ability to remain safe in the human body for controlled lengths of time makes biodegradable polymers an ideal material for medical applications, representing an alternative to traditional polymers. Developments of newer devices focus on attaining optimal antimicrobial efficacy with minimal systemic drug concentration and toxicity. Yu et $\mathrm{a}^{26}$ has successfully coated polyacrylonitrile nanofibers with 
silver nanoparticles, using a modified coaxial electrospinning process, and demonstrated excellent antimicrobial activities against Bacillus subtilis and Escherichia coli. In the present study, we specifically selected vancomycin as the carried drug because vancomycin has been the mainstay for vascular prosthetic infection. Biodegradable polymers offer controlled elution of active drugs from the vascular prosthetic grafts through means of biocompatible polymer nanofibers. After completion of their useful function, the nanofibers degrade slowly to inert organic monomers, subsequently dissipating the risk associated with the long-term presence of durable polymers in the tissues. PLGA belongs to a class of synthesized biodegradable and biocompatible copolymers, from which resorbable sutures, resorbable surgical clips, and controlled-release implants are made. PLGA also falls within the class of copolymers that have been used for implantable and injectable controlled-release, drug delivery systems. These copolymers, with a history of safe use, have been approved for human use. Following its introduction into the human body, PLGA material induces only a minimal inflammatory response and biodegrades through the hydrolysis of its ester linkages to yield biocompatible lactic and glycolic acids. ${ }^{27}$ It has been shown that the time required for degradation of PLGA is related to the monomer ratio used in production: the higher the content of glycolide units, the lower the time required for degradation as compared with predominantly lactide materials. An exception to this rule is the copolymer with a 50:50 monomer ratio, which exhibits the faster degradation (about 2 months). This unique characteristic makes PLGA an ideal material as a carrier for drug delivery.

Compared with other slow-release mechanisms, drugloaded nanofibers, due to their small diameter distributions, can provide short diffusion passage length. The high surface area also facilitates mass transfer and effective drug release. In addition, the drug-loaded nanofibers derived from electrospinning have, not only one dimension at the microscopic scale, but another dimension in the macroscopic form. This unique characteristic endows the electrospun nanofibers with the merits possessed by the nanosized drug delivery system in altering the biopharmaceutic and pharmacokinetic properties of the drug molecule for favorable clinical outcomes, as well as the advantages of conventional solid dosage forms, such as easy processing, good drug stability, and ease of packaging and shipping. ${ }^{28}$ Previous studies reported that the electrospinning process might denature the biological and structural properties of a natural protein such as collagen. ${ }^{29}$ The experimental results of this study demonstrate that the PLGA/vancomycin nanofibers remain intact during the processing procedure and can function as an excellent vehicle for drug delivery. Furthermore, the function of antimicrobial vancomycin remains high after the electrospinning process. The findings in this study suggest that electrospun drugeluting PLGA/vancomycin nanofibers may be a promising candidate for antibiotic release, to assist the treatment of vascular prosthesis infection and to prevent reinfection.

Despite its contributions, this study has several limitations. First, the assessment of sustained vancomycin elution via biodegradable nanofiber-loaded grafts was carried out in a noninfected animal model. Whether or not the drug-eluting prosthetic grafts will perform differently in an infected space is unknown. Further evaluation of the antibiotic-loaded grafts in an infection model is necessary to answer this question. Second, the vascular grafts were implanted into the subcutaneous pocket but were not anastomosed to the blood vessels as in the clinical scenario. How blood flow might affect the results of this study is unknown. If a considerable amount of vancomycin were released to the graft or to the inside of the graft, the serum vancomycin level might be increased. Third, current animal models used in assessing the performance of drug-eluting grafts are limited with respect to the ability to replicate human conditions, although results with the rabbit model have been generally representative of human responses, with a different timeline of healing. These will be the topics of our future works.

\section{Conclusion}

This study developed biodegradable drug-eluting nanofibers from PLGA materials, produced by the electrospinning process, for vascular prosthetic graft-related applications. The in vitro release rates of the pharmaceutical from the nanofibers was characterized using an elution method and an HPLC assay. Experimental results indicated that the biodegradable nanofibers released high concentrations of vancomycin in vitro for 32 days. Additionally, the animal study of the drugeluting grafts in the subcutaneous tissue of rabbits suggested that the nanofibers could have an in vivo release of high concentrations of vancomycin, well above the $\mathrm{MIC}_{90}$, for 35 days. Most importantly, the drug-eluting grafts developed in this work have allowed us to achieve local and sustainable delivery of antimicrobial agents. The new drug-eluting graft has the potential to be of use to resist future infections when an infected graft is to be exchanged.

\section{Acknowledgment}

The authors would like to thank Chang Gung Memorial Hospital (Linkou, Taiwan) for financially supporting this research, under contract number CMRPD3D0141. 


\section{Disclosure}

The authors report no conflicts of interest in this work.

\section{References}

1. Ryan SV, Calligaro KD, Scharff J, Dougherty MJ. Management of infected prosthetic dialysis arteriovenous grafts. J Vasc Surg. 2004; 39(1):73-78.

2. Liu C, Bayer A, Cosgrove SE, et al; Infectious Diseases Society of America. Clinical practice guidelines by the Infectious Diseases Society of America for the treatment of methicillin-resistant Staphylococcus aureus infections in adults and children. Clin Infect Dis. 2011;52(3): e18-e55.

3. Peng CW, Tan SG. Polyurethane grafts: a viable alternative for dialysis arteriovenous access? Asian Cardiovasc Thorac Ann. 2003;11(4): 314-318.

4. Englesbe MJ, Al-Holou WN, Moyer AT, et al. Single center review of femoral arteriovenous grafts for hemodialysis. World J Surg. 2006; 30(2):171-175.

5. Schutte WP, Helmer SD, Salazar L, Smith JL. Surgical treatment of infected prosthetic dialysis arteriovenous grafts: total versus partial graft excision. Am J Surg. 2007;193(3):385-388; discussion 388.

6. Bachleda P, Kalinova L, Utikal P, Kolar M, Hricova K, Stosova T. Infected prosthetic dialysis arteriovenous grafts: a single dialysis center study. Surg Infect (Larchmt). 2012;13(6):366-370.

7. Stone PA, Mousa AY, Hass SM, et al. Antibiotic-loaded polymethylmethacrylate beads for the treatment of extracavitary vascular surgical site infections. J Vasc Surg. 2012;55(6):1706-1711.

8. Cosgrove SE, Sakoulas G, Perencevich EN, Schwaber MJ, Karchmer AW, Carmeli Y. Comparison of mortality associated with methicillin-resistant and methicillin-susceptible Staphylococcus aureus bacteremia: a metaanalysis. Clin Infect Dis. 2003;36(1):53-59.

9. Whitby M, McLaws ML, Berry G. Risk of death from methicillinresistant Staphylococcus aureus bacteraemia: a meta-analysis. Med J Aust. 2001;175(5):264-267.

10. Kang CI, Song JH, Chung DR, et al; Asian Network for Surveillance of Resistant Pathogens (ANSORP) Study Group. Clinical impact of methicillin resistance on outcome of patients with Staphylococcus aureus infection: a stratified analysis according to underlying diseases and sites of infection in a large prospective cohort. J Infect. 2010;61(4): 299-306.

11. Yaw LK, Robinson JO, Ho KM. A comparison of long-term outcomes after meticillin-resistant and meticillin-sensitive Staphylococcus aureus bacteraemia: an observational cohort study. Lancet Infect Dis. 2014; 14(10):967-975.

12. Gould FK, Brindle R, Chadwick PR, et al; MRSA Working Party of the British Society for Antimicrobial Chemotherapy. Guidelines (2008) for the prophylaxis and treatment of methicillin-resistant Staphylococcus aureus (MRSA) infections in the United Kingdom. J Antimicrob Chemother. 2009;63(5):849-861.

13. Hidayat LK, Hsu DI, Quist R, Shriner KA, Wong-Beringer A. Highdose vancomycin therapy for methicillin-resistant Staphylococcus aureus infections: efficacy and toxicity. Arch Intern Med. 2006;166(19): 2138-2144.
14. Jeffres MN, Isakow W, Doherty JA, Micek ST, Kollef MH. A retrospective analysis of possible renal toxicity associated with vancomycin in patients with health care-associated methicillin-resistant Staphylococcus aureus pneumonia. Clin Ther. 2007;29(6):1107-1115.

15. Forouzesh A, Moise PA, Sakoulas G. Vancomycin ototoxicity: a reevaluation in an era of increasing doses. Antimicrob Agents Chemother. 2009;53(2):483-486.

16. Sacar M, Sacar S, Kaleli I, et al. Linezolid alone and in combination with rifampicin prevents experimental vascular graft infection due to methicillin-resistant Staphylococcus aureus and Staphylococcus epidermidis. J Surg Res. 2007;139(2):170-175.

17. Koshiko S, Sasajima T, Muraki S, et al. Limitations in the use of rifampicin-gelatin grafts against virulent organisms. J Vasc Surg. 2002; 35(4):779-785.

18. Stewart AH, Eyers PS, Earnshaw JJ. Prevention of infection in peripheral arterial reconstruction: a systematic review and meta-analysis J Vasc Surg. 2007;46(1):148-155.

19. Fujimoto K, Yamamura K, Osada T, et al. Subcutaneous tissue distribution of vancomycin from a fibrin glue/Dacron graft carrier. J Biomed Mater Res. 1997;36(4):564-567.

20. Morishima M, Marui A, Yanagi S, et al. Sustained release of vancomycin from a new biodegradable glue to prevent methicillin-resistant Staphylococcus aureus graft infection. Interact Cardiovasc Thorac Surg. 2010;11(1):52-55.

21. Yu DG, Chian W, Wang X, Li XY, Li Y, Liao YZ. Linear drug release membrane prepared by a modified coaxial electrospinning process. J Membrane Sci. 2013;428:150-156.

22. Yu DG, Li XY, Wang X, Chian W, Liao YZ, Li Y. Zero-order drug release cellulose acetate nanofibers prepared using coaxial electrospinning. Cellulose. 2013;20:379-389.

23. Liu KS, Liu SJ, Chen HY, et al. Steady antibiotic release from biodegradable beads in the pleural cavity: an in vitro and in vivo study Chest. 2012;141(5):1197-1202.

24. Chen DW, Liao JY, Liu SJ, Chan EC. Novel biodegradable sandwichstructured nanofibrous drug-eluting membranes for repair of infected wounds: an in vitro and in vivo study. Int J Nanomedicine. 2012;7: 763-771.

25. Appleman MD, Citron DM. Efficacy of vancomycin and daptomycin against Staphylococcus aureus isolates collected over 29 years. Diagn Microbiol Infect Dis. 2010;66(4):441-444.

26. Yu DG, Zhou J, Chatterton NP, Li Y, Huang J, Wang X. Polyacrylonitrile nanofibers coated with silver nanoparticles using a modified coaxial electrospinning process. Int J Nanomedicine. 2012;7:5725-5732.

27. Middleton JC, Tipton AJ. Synthetic biodegradable polymers as orthopedic devices. Biomaterials. 2000;21(23):2335-2346.

28. Yu DG, Zhu LM, White K, Branford-White C. Electrospun nanofiberbased drug delivery systems. Health. 2009;1(2):67-75.

29. Kumbar SG, Nukavarapu SP, James R, Nair LS, Laurencin CT Electrospun poly(lactic acid-co-glycolic acid) scaffolds for skin tissue engineering. Biomaterials. 2008;29(30):4100-4107.
International Journal of Nanomedicine

\section{Publish your work in this journal}

The International Journal of Nanomedicine is an international, peerreviewed journal focusing on the application of nanotechnology in diagnostics, therapeutics, and drug delivery systems throughou the biomedical field. This journal is indexed on PubMed Central, MedLine, CAS, SciSearch ${ }^{\circledR}$, Current Contents ${ }^{\circledR} /$ Clinical Medicine,

\section{Dovepress}

Journal Citation Reports/Science Edition, EMBase, Scopus and the Elsevier Bibliographic databases. The manuscript management system is completely online and includes a very quick and fair peer-review system, which is all easy to use. Visit http://www.dovepress.com/ testimonials.php to read real quotes from published authors. 